\title{
Multifocal strokes in a 56-year-old man with HIV infection
}

\author{
Ariel Lefkowitz MDCM, Wayne L. Gold MD, Leanne K. Casaubon MD MSc, Daniel M. Mandell MD PhD, \\ Jeffrey Craig MD
}

A 56-year-old man presented with a oneday history of vomiting, vertigo and blurred vision in both eyes preceded by a three-week history of fever, night sweats and a $5-\mathrm{kg}$ weight loss. He had no recent history of travel or contact with sick people. His past medical history was notable for untreated HIV infection diagnosed eight months previously, after treatment for presumed Pneumocystis jirovecii pneumonia. At that time, his CD4 count was $0.072 \times 10^{9} / \mathrm{L}$. He was immune to hepatitis B virus infection secondary to a previous natural infection, and he had remote idiopathic retinal vasculitis. He had had chickenpox as a child. There was no history of hypertension, diabetes mellitus, smoking, dyslipidemia, atrial fibrillation or cardiac disease. He denied intravenous drug use.

On examination, the patient was afebrile, his blood pressure was $133 / 83 \mathrm{~mm} \mathrm{Hg}$, and his heart rate was 57 beats/min and regular. He was somnolent but rousable and answered questions appropriately. Findings on cardiovascular, respiratory and abdominal examinations were normal. Neurologic examination showed bilateral eye deviation to the right as well as horizontal, vertical and torsional nystagmus in primary position, which worsened with rightward gaze. There was right- sided miosis but no ptosis. Speech and swallowing were normal. Muscle tone, power and reflexes were normal. There was decreased sensation to pinprick in the right cheek and the left arm and leg. The patient had marked ataxia in his right arm and leg (appendicular ataxia). Dermatologic examination revealed several crusted skin lesions distributed over his body, with a single vesicle overlying his right zygoma. There were no peripheral findings of infective endocarditis.

The patient's leukocyte count was 2.4 (normal 4.0-11.0) $\times 10^{9} / \mathrm{L}$, hemoglobin $\mathrm{A}_{1 \mathrm{C}}$ concentration $6 \%$ (normal 4\%-6\%) and low-density lipoprotein cholesterol $2.76 \mathrm{mmol} / \mathrm{L}$. Blood cultures for bacteria were negative. Tests of the patient's serum were negative for syphilis and cryptococcal antigen. The erythrocyte sedimentation rate was 41 (normal 0-10) $\mathrm{mm} / \mathrm{h}$.

Magnetic resonance imaging of the brain showed small acute infarcts in the left frontal cortex and deep white matter (the territory supplied by the left middle cerebral artery) and in the right lateral medulla and right cerebellar hemisphere (the territory supplied by the right posterior inferior cerebellar artery) (Figure 1). Computed tomography (CT) angiography showed smooth narrowing of the left posterior cerebral artery with

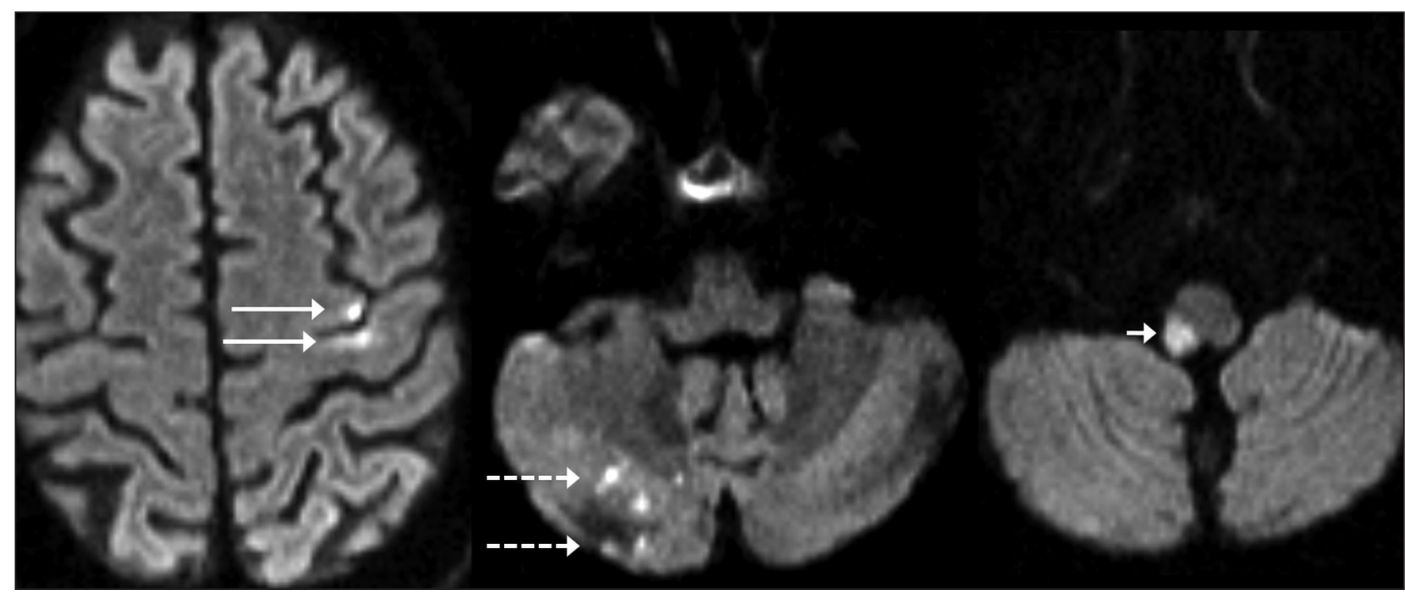

Figure 1: Diffusion-weighted magnetic resonance imaging in the axial plane showing acute infarcts in the left frontal cortex (solid arrow), right cerebellar hemisphere (dotted arrow) and right lateral medulla (short arrow) in a 56-year-old man with HIV infection who presented with multifocal strokes. 
post-stenotic dilatation and smooth narrowing of the superior cerebellar arteries (Figure 2), as well as narrowing of the right posterior inferior cerebellar artery (not shown). The aortic arch and neck arteries were normal on angiography. Electrocardiography showed normal sinus rhythm, and results of a subsequent 48-hour Holter monitor did not show atrial fibrillation. A transthoracic echocardiogram appeared normal.

\section{What is the most likely cause of the patient's strokes?}

a. Varicella-zoster virus (VZV) vasculopathy in the central nervous system (CNS)

b. Cardioembolic ischemic strokes secondary to atrial fibrillation

c. Large-artery embolic strokes from the great vessels of the neck

d. Meningovascular syphilis

We found evidence of infarctions in multiple vascular territories in this patient. The rightsided miosis, nystagmus and incoordination,

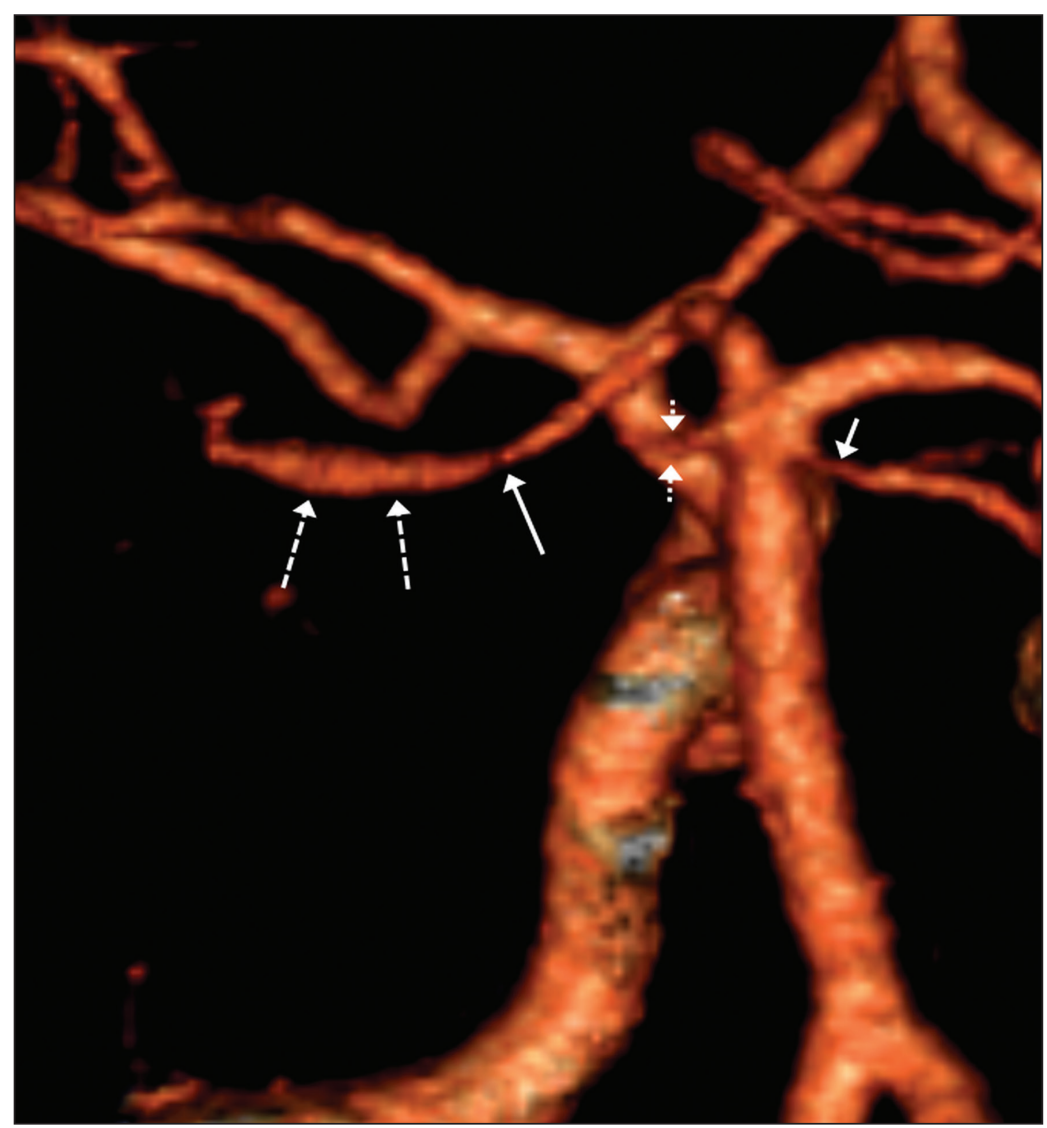

Figure 2: Computed tomography angiography showing smooth narrowing of the left posterior cerebral artery (long solid arrow) with post-stenotic dilatation (long dotted arrows), severe narrowing of the left superior cerebellar artery (short dotted arrows) and moderate narrowing of the right superior cerebellar artery (short solid arrow). along with sensory deficits involving the right side of his face and left limbs suggest a partial right lateral medullary syndrome compatible with the imaging findings. The right cerebellar infarction may have contributed to his ataxia, whereas the left frontal infarction was small and likely asymptomatic.

Common causes of ischemic stroke in adults include cardioembolism due to thromboembolism from large-artery atherosclerosis and atrial fibrillation. Infarcts in multiple vascular territories are consistent with a cardioembolic mechanism. Although the patient's echocardiogram, electrocardiogram and Holter monitor results were normal, paroxysmal atrial fibrillation is possible, which may be identified with prolonged electrophysiologic monitoring. ${ }^{1}$ Although embolism from the neck arteries may cause stroke, simultaneous infarctions in the anterior and posterior circulation make this mechanism unlikely. Furthermore, the smooth narrowing of intracranial arteries is not typical of thromboembolic narrowing or intracranial atherosclerosis. The appearance on CT angiography is more suggestive of an inflammatory arteriopathy.

Meningovascular syphilis, a form of tertiary syphilis, can present with multi-territorial strokes and is well-described in patients with HIV infection $^{2}$ (Box $1^{3}$ ). Skin manifestations of syphilis, however, are typically associated with the secondary stage of the disease and are not vesicular. Furthermore, this patient's serum syphilis screen was negative.

The presence of crusting skin lesions and an active vesicle raise suspicion of VZV infection. The patient's prodromal symptoms, multifocal strokes and multifocal smooth narrowing of intracranial arteries seen on CT angiography make (a) VZV CNS vasculopathy a likely diagnosis.

\section{How can the diagnosis of VZV CNS vasculopathy be confirmed?}

a. Detection of VZV by polymerase chain reaction (PCR) from an unroofed vesicle

b. Detection of VZV by PCR in a sample of cerebrospinal fluid

c. Measurement of immunoglobulins $\mathrm{G}$ and $\mathrm{M}$ against VZV in a blood sample

d. Biopsy of the cerebral artery wall, with pathological examination and detection of VZV by PCR

Both (a) and (b) are appropriate diagnostic tests. Despite clinical evidence of vesicles and radiologic features of stroke, virologic confirmation is required for the diagnosis of VZV vasculopathy in 
the CNS. A positive PCR result from an unroofed vesicle will establish the diagnosis of VZV; however, detection of VZV from a sample of cerebrospinal fluid provides strong evidence in support of CNS involvement. ${ }^{4}$ Positive results of VZV blood serology will not confirm CNS involvement; an isolated elevated IgG titer would be compatible with the patient's remote history of chickenpox. Biopsy of the cerebral artery wall has not been established as a gold standard for the diagnosis of VZV CNS vasculopathy and would expose the patient to unnecessary operative risk. ${ }^{4}$

In this patient, PCR of the cerebrospinal fluid and the unroofed vesicle were performed and VZV was detected. Results of the cerebrospinal fluid analysis are summarized in Box 2 .

\section{What treatment should be prescribed for this patient?}
a. Oral acyclovir
b. Oral valacyclovir
c. Intravenous acyclovir
d. Intravenous acyclovir and oral prednisone

There is little evidence to support specific treatment regimens for VZV CNS vasculopathy. Successful treatment of CNS infection requires adequate drug levels in the cerebrospinal fluid and brain parenchyma. For VZV vasculopathy, this can optimally be achieved with (c) intravenous acyclovir. This choice is recommended by most experts, at the dose typically used for the treatment of encephalitis due to herpes simplex virus (10-15 mg/kg every $8 \mathrm{~h}$, assuming normal renal function). ${ }^{4}$ Oral use of valacyclovir has not been fully studied in this setting, and to our knowledge its use as primary therapy has not been reported in the literature. Evidence for the addition of corticosteroids for treatment of this condition is in the form of case reports and expert opinion.

\section{Discussion}

A serious neurologic complication of VZV reactivation, VZV CNS vasculopathy may occur in both immunocompromised and immunocompetent patients. ${ }^{5}$ The incidence and prevalence of VZV vasculopathy in the general population is not known, with data limited to case reports. ${ }^{6}$ The largest case series found that $10 \%$ of patients were HIV positive. ${ }^{6}$

With reactivation, VZV spreads centripetally from the dorsal root ganglia along the axon, with transmural migration to blood vessels of the CNS. $^{7}$ Infection and inflammation of the vessels can result in ischemic stroke by narrowing the arterial lumen or inducing arterial thrombosis. ${ }^{8}$

Patients frequently present with one or more of focal neurologic deficits, subacute onset of headache or changes in mental status. In a case series of 30 patients with VZV CNS vasculopathy, only $63 \%$ had rash at the time of diagnosis. ${ }^{6}$ In those with rash at any time during their illness, the mean time from onset of rash to the development of symptoms was 4.1 months. ${ }^{6}$ The absence of rash at the time of neurologic involvement may make the diagnosis challenging, because a history of varicella zoster in the months before presentation may not be elicited. Furthermore, VZV reactivation can occur in the absence of rash (zoster sine herpete), which makes diagnosis even more challenging. ${ }^{9}$

VZV CNS vasculopathy can affect the large arteries of the circle of Willis but also the smaller intracranial arteries. In some patients, there is a mixed pattern. Arterial narrowing can be unifocal or multifocal, and on imaging, it usually looks like the type of smooth narrowing seen in other vasculitic conditions. ${ }^{6}$ Lack of pleocytosis in the cerebrospinal fluid may be seen in up to $33 \%$ of

Box 1: Infectious pathogens that are associated with or are probable causes of stroke ${ }^{3}$

\begin{tabular}{|c|c|}
\hline Pathogen & Mechanism of stroke \\
\hline \multicolumn{2}{|l|}{ Bacteria } \\
\hline $\begin{array}{l}\text { Agents of infective } \\
\text { endocarditis* }\end{array}$ & Cardioembolic \\
\hline Streptococcus pneumoniae & $\begin{array}{l}\text { Vasculitis secondary to bacterial meningitis; } \\
\text { contiguous inflammation of vessel from } \\
\text { paranasal sinus infection }\end{array}$ \\
\hline Treponema pallidum & $\begin{array}{l}\text { Endarteritis obliterans secondary to } \\
\text { meningovascular syphilis }\end{array}$ \\
\hline \multicolumn{2}{|l|}{ Mycobacteria } \\
\hline Mycobacterium tuberculosis & Vasculitis secondary to tuberculous meningitis \\
\hline \multicolumn{2}{|l|}{ Fungi } \\
\hline Cryptococcus neoformans & Vasculitis secondary to cryptococcal meningitis \\
\hline Aspergillus species & $\begin{array}{l}\text { Hematogenous dissemination from } \\
\text { extracranial sites of infection; angioinvasion } \\
\text { from contiguous rhino-sino-orbital infection }\end{array}$ \\
\hline Agents of mucormycosis & $\begin{array}{l}\text { Angioinvasion from contiguous rhino-sino- } \\
\text { orbital infection }\end{array}$ \\
\hline \multicolumn{2}{|l|}{ Viruses } \\
\hline Varicella-zoster virus & Vasculitis or vasculopathy \\
\hline Cytomegalovirus & Vasculitis or vasculopathy \\
\hline HIV & Vasculopathy \\
\hline \multicolumn{2}{|l|}{ Parasites } \\
\hline Plasmodium falciparum & $\begin{array}{l}\text { Sequestration of parasitized erythrocytes in the } \\
\text { cerebral microvasculature in cerebral malaria }\end{array}$ \\
\hline
\end{tabular}


patients, ${ }^{6}$ including those who are immunocompromised, such as our patient.

The current literature suggests an increased risk of stroke in the short term following VZV reactivation. A recent self-controlled case series of 6584 individuals showed that the rate of stroke was increased in the first four weeks following herpes zoster (age-adjusted incidence ratio 1.63, 95\% confidence interval 1.32-2.02), with continued risk persisting to six months, compared with the rate in the six months before the development of herpes zoster. ${ }^{10}$ Rates of stroke were higher among patients with zoster ophthalmicus, those with trigeminal nerve involvement and patients who did not receive oral antiviral treatment.

VZV CNS vasculopathy is a treatable infectious cause of stroke (Box 1). Intravenous acyclovir treatment is recommended; however, the optimal duration is not known. Expert opinion recommends a 14-day course in immunocompetent patients, and a longer course in immunocompromised patients, particularly if they have persistent neurologic symptoms, where expert opinion recommends oral valacyclovir treatment for one to two additional months. ${ }^{4}$

Because the onset of symptoms of VZV vasculopathy in many patients is remote from the episode of VZV infection, the host inflammatory response may in part be responsible for the injury that occurs to the blood vessels. For this reason, some experts have recommended the addition of corticosteroid treatment to control inflammation of vessel walls. This recommendation is controversial. In a small case series of patients given acyclovir, $75 \%$ had an improved or stable condition when corticosteroid treatment was added, as compared with $66 \%$ who did not receive corticosteroids. ${ }^{6}$ In this series, however, the setting was uncontrolled, and treatment regimens differed. Further prospective studies are needed before the

Box 2: Results of cerebrospinal fluid analysis

\begin{tabular}{|lc|}
\hline Investigation & Result \\
\hline Leukocyte count, $\times 10^{6} / \mathrm{L}$ (normal 0-5) & 0 \\
\hline Glucose level, mmol/L (normal 2.2-4.4) & 2.8 \\
\hline Protein level, g/L (normal 0.45) & 0.48 \\
\hline Bacterial culture & Negative \\
\hline Fungal culture & Negative \\
\hline Cryptococcal antigen & Negative \\
\hline Syphilis screen (VDRL and FTA-ABS) & Negative \\
\hline Varicella-zoster virus PCR & Positive \\
\hline $\begin{array}{l}\text { Note: FTA-ABS = fluorescent treponemal antibody absorption, PCR = polymerase chain } \\
\text { reaction, VDRL = Venereal Disease Reference Laboratory. }\end{array}$ \\
\hline
\end{tabular}

addition of corticosteroid treatment becomes the standard of care for this condition. In the meantime, the decision to add corticosteroid treatment should be made on an individual basis in consultation with an infectious diseases specialist.

Given the positive PCR result for VZV in the cerebrospinal fluid, neuroimaging findings compatible with vasculopathy in the CNS and the absence of an alternative cause of stroke in our patient, we diagnosed VZV vasculopathy and prescribed a 21-day course of intravenous acyclovir treatment at a dose of $10 \mathrm{mg} / \mathrm{kg}$ every eight hours. We did not add corticosteroid treatment because of the paucity of data in patients with advanced HIV infection. The patient was discharged from hospital to a stroke rehabilitation centre, where his neurologic deficits gradually improved. He was subsequently prescribed highly active antiretroviral therapy.

This case highlights the important role of infections in the differential diagnosis of multifocal ischemic strokes, particularly in immunocompromised people. The diagnosis of VZV CNS vasculopathy may be particularly challenging when cutaneous features of varicella zoster are absent at the time of neurologic presentation.

\section{References}

1. Gladstone DJ, Spring M, Dorian P, et al. Atrial fibrillation in patients with cryptogenic stroke. N Engl J Med 2014;370:2467-77.

2. Peters M, Gottschalk D, Boit R, et al. Meningovascular neurosyphilis in human immunodeficiency virus infection as a differential diagnosis of focal CNS lesions: a clinicopathological study. J Infect 1993;27:57-62.

3. Fugate JE, Lyons JL, Thakur KT, et al. Infectious causes of stroke. Lancet Infect Dis 2014;14:869-80.

4. Gilden D, Cohrs RJ, Mahalingam R, et al. Varicella zoster virus vasculopathies: diverse clinical manifestations, laboratory features, pathogenesis, and treatment. Lancet Neurol 2009;8:731-40.

5. Mareedu J, Hanumaiah RG, Hale E, et al. Varicella zoster vasculopathy. J Int Assoc Physicians AIDS Care (Chic) 2011;10:144-5.

6. Nagel MA, Cohrs RJ, Mahalingam R, et al. The varicella zoster virus vasculopathies: clinical, CSF, imaging, and virologic features. Neurology 2008;70:853-60.

7. Nagel MA, Traktinskiy I, Azarkh Y, et al. Varicella zoster virus vasculopathy: analysis of virus-infected arteries. Neurology 2011; 77:364-70.

8. Mueller NH, Gilden DH, Cohrs RJ, et al. Varicella zoster virus infection: clinical features, molecular pathogenesis of disease, and latency. Neurol Clin 2008;26:675-97.

9. Gilden DH, Dueland AN, Devlin ME, et al. Varicella-zoster virus reactivation without rash. J Infect Dis 1992;166(Suppl 1):S30-4.

10. Langan SM, Minassian C, Smeeth L, et al. Risk of stroke following herpes zoster: a self-controlled case-series study. Clin Infect Dis 2014;58:1497-503.

Affiliations: Department of Medicine (Lefkowitz, Gold, Craig), University of Toronto; Divisions of Infectious Diseases (Gold, Craig), Neurology (Casaubon) and Neuroradiology (Mandell), University Health Network, Toronto, Ont.

Contributors: Ariel Lefkowitz, Wayne Gold, Daniel Mandell and Jeffrey Craig were involved in the clinical care of the patient. All of the authors were involved in the conception of the report and the literature review. Ariel Lefkowitz wrote the initial draft of the manuscript. All of the authors contributed substantially to revising of the manuscript, approved the final version to be published and agreed to act as guarantors of the work. 